\title{
Immuno-pathophysiological responses of mouse model to experimental infection with Brucella melitensis and its lipopolysaccharides via intraperitoneal route
}

\begin{abstract}
Brucella melitensis is one of the major zoonotic pathogens with significant economic implications worldwide. The pathogenicity is complex and not always well understood. Lipopolysaccharide (LPS) remains the major virulent factor of B. melitensis and responsible for the mechanism by which the pathogen causes its deleterious effects. In this study, 84 mice of 6-8 weeks old of both sexes were divided equally into 3 groups; namely Brucella melitensis infected group, lipopolysaccharide (LPS) infected group and control group. The former two groups contained 36 mice each with equal gender distribution. The control group consisted of 12 mice only. Animals in B. melitensis infected group, a single inoculum of $0.4 \mathrm{ml}$ containing $10^{9}$ of B. melitensis were intraperitoneally challenged while animals in LPS group, a single dose of $0.4 \mathrm{ml}$ containing LPS extracted from the B. melitensis were intraperitoneally inoculated. Animals in control group received intraperitoneally, a single dose of $0.4 \mathrm{ml}$ phosphate buffered saline (PBS) of $\mathrm{pH} 7$. Animals that were infected intraperitoneally with B. melitensis demonstrated significant clinical presentation; gross and histo-pathological evidence than LPS infected group. However, both infected groups showed elevated levels of interleukins (IL-1 $\beta$ and IL6), antibody levels (IgM an IgG) as early as 3 days post-infection with predominance in LPS infected group. In contrast, low levels of sex related hormonal changes in which LPS infected group showed the least concentration were also detected throughout the experimental period. In conclusion, B. melitensis can be transmitted via gastrointestinal, respiratory and reproductive tract. Moreover, LPS stimulated significantly the innate and acquired immune system without significant systemic dysfunction, suggesting potentiality of the protective properties of this component as alternative vaccine for brucellosis infection.
\end{abstract}

Keyword: Brucella melitensis; Lipopolysaccharide; Mouse model; Intraperitoneal route of infection; Clinico-pathological; Histopathology; Cytokine; Antibody; Hormone; PCR 